Copyright (C) 2003 IEEE. Reprinted from Engineering in Medicine and Biology Society, 2003. Proceedings of the 25th Annual International Conference of the IEEE , vol.1, no., pp. 694- 697 Vol.1, 17-21 Sept. 2003

This material is posted here with permission of the IEEE. Internal or personal use of this material is permitted. However, permission to reprint/republish this material for advertising or promotional purposes or for creating new collective works for resale or redistribution must be obtained from the IEEE by writing to pubs-permissions@ieee.org. By choosing to view this document, you agree to all provisions of the copyright laws protecting it. 


\title{
An efficient multiscale approach to level set evolution
}

\author{
Hongmei Zhang ${ }^{1}$ Zhengzhong Bian ${ }^{1}$ Youmin Guo ${ }^{2}$ Baowei $\mathrm{Fei}^{1} \mathrm{Min} \mathrm{Ye}^{3}$. \\ ${ }^{1}$ (School of Life Science and Technology, Xi'an Jiaotong University, Xi'an 710049, China) \\ ${ }^{2}$ (First affiliated hospital, Xi' an Jiaotong University, Xi' an 710049, China) \\ ${ }^{3}$ (Construction Machinery College, Chang'an University, Xi'an 710064, China)
}

\begin{abstract}
In this paper, an efficient multiscale scheme for level set evolution is proposed. First, we are addressing the problem of passing the solution from the coarser scale to the finer one. Inspired by the idea of the entropy condition and its extention, an efficient passing solution method is presented, where neither extrapolation nor complex computation is needed. Thus it could induce fast convergence rate. Furthermore, an improved Hermes algorithm, called fast Hermes, is developed to fast implement the level set evolution on each scale by further loosening the constraint in the intermediate levels. Our approach is evaluated and compared to the existing algorithm. The experimental results are very promising.
\end{abstract}

Keywords-Level set, front propagation, multiscale, potential front, fast Hermes, segmentation

\section{INTRODUCTION}

Level set method for front propagation was first introduced by Osher and Sethian[1]. It offers a highly robust mathematical and numerical implementation on curve / surface evolution. Its major virtue is that it naturally constructs the fundamental weak solution to interface propagation posed by Sethian. The essential idea of the level set method is to view the moving front as the zero level set of a higher dimensional function. Thus, topological changes of the moving front can be handled naturally by exploiting the zero level set.

Efficient schemes for numerical implementation of the level set evolution have been extensively exploited. The widely used two are Narrow Band method and Fast Marching method. Fast Marching algorithm is a very fast numerical scheme for level set evolution, but it could only handle the monotonically advancing front problem that requires the speed function is always positive or negative. Narrow Band method could deal with all types of speed function problem, whereas its computational cost is very expensive. Hermes algorithm is proposed by Nikos in [2], which combines the Narrow Band and Fast Marching method by employing a selective propagation over a relatively small window. The advantages of this approach lie in that its computational cost is comparable to Fast Marching approach and that it can deal with all types of curve propagation as the Narrow Band method.

Sophisticated methodology is to implement the above algorithm on a multiresolution space. Using a coarse to fine pyramid, the final solution on the coarser scale is used to determine the initial front condition on the finer scale. This approach is applied in [3] where an extrapolation of the solution from the level with low resolution to the level with finer solution configurations takes place.

In this paper, we propose a new multiscale scheme for the level set evolution. First, borrowed from the entropy condition and extend it, an efficient approach to pass the solution from the coarser scale to the finer one is presented, where neither extrapolation nor complex computation is needed that could induce fast convergence rate. Second, An improved Hermes algorithm, we call it fast Hermes, is developed to fast implement the level set evolution numerically on each scale.

In section II, level set method is briefly outlined; in section III, multiscale scheme for the level set evolution is described in detail, where a new passing solution method and fast Hermes algorithm is developed; in section IV, experimental results by our approach is presented and compared to the existing algorithm; finally in section $\mathrm{V}$, conclusions are reported.

\section{LEVEL SET METHOD}

The essential idea of the level set method is to embed the moving front as zero level set of a higher dimensional function. Let $C(P):[0,1] \rightarrow R^{2}$ be a closed parameterized curve in Euclidean plane $R^{2}$. Let $\phi: R^{2} \rightarrow R$ be the signed distance function to curve $C, C$ is thus the zero level set of $\phi$ and $\phi$ is an implicit representation of $C$. Let $C_{0}$ be the initial curve, $\phi_{0}$ be the signed distance function to $C_{0}$. As previously reported [1], evolving $C$ according to:

$$
\left\{\begin{array}{l}
\frac{\partial C}{\partial t}=F \cdot \vec{N} \\
C(p, 0)=C_{0}(p)
\end{array}\right.
$$

is equivalent to evolve $\phi$ by:

$$
\left\{\begin{array}{l}
\phi_{l}+F \cdot|\nabla \phi|=0 \\
\phi(\cdot, 0)=\phi_{0}(\cdot) \text { and } \phi_{0}\left(C_{0}\right)=0 \text { given }
\end{array}\right.
$$

where $F$ is the speed function and $\vec{N}$ is the normal vector given by $\vec{N}=\frac{\nabla \phi}{|\nabla \phi|}$.

\section{MULTISCALE LEVEL SET EVOLUTION SCHEME}

Let $I_{0} I_{1}, \ldots, I_{J}$ be a set of multiscale image set, $I_{J}$ the coarsest scale image and $I_{0}$ the original image. Multiscale scheme for exploiting the solution of (2) is obtained by 
coarse to fine performing the following level set evolution equations:

$$
\begin{aligned}
& \phi_{t}+F\left(I_{J}\right) \cdot|\nabla \phi|=0 \quad \text { given the initial curve } \\
& \left\{\begin{array}{l}
\phi_{t}+F\left(I_{j}\right) \cdot|\nabla \phi|=0 \\
\phi(\cdot, 0) \quad \text { given intial front condition by passing solution }
\end{array}\right.
\end{aligned}
$$

\section{A. Passing the solution from coarser scale to finer scale}

Nikos et al [3] proposed a multiscale level set evolution scheme where extrapolation is used to pass the solution from the coarser scale to the finer one. In this section, a new efficient passing solution method is presented, where neither extrapolation nor complex computation is needed.

The level set method constructs the entropy satisfactory weak solution. The entropy condition may be stated as follows: once a particle is burned, it stays burnt [4]. The entropy condition allows us to determine the boundary of the propagating front. It could be considered as a flame separating a burnt region from an unburnt region.

Borrowed from this idea, the current front can always be viewed as the propagating points ("flaming") that separate the burnt region ("flamed") from the unburnt region ("unflamed"). The image pixels are classified into three distinct categories:

"flaming pixels": the pixels that belong to the current front; "flamed pixels": the pixels that belong to the previous front but not the current one;

"unflamed pixels": the pixels that have never been reached by the front;

Assume that if a "parent" on the coarser scale is the "flamed" point then its four children on the finer scale are also the "flamed" points. So the "flamed region" on the finer scale $j$ is the children of all "flamed parent" on the $j+1$ th scale. The potential front on the $j$ th scale should be the boundary separating the "flamed region" from "unflamed region", that is, also to be all the "unflamed neighbors" of the "flamed children" (Fig 1). The obtained new front on the finer scale is almost the same topological shape as that of the coarser scale.

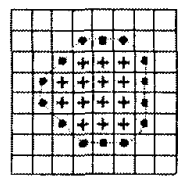

$j+1$ th scale

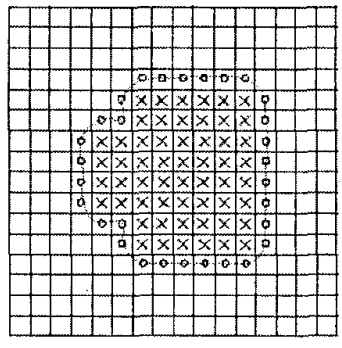

$j$ th scale
Fig 1 passing the solution from the coarser scale to the finer one

As shown in fig 1 , the current front on the $j+1$ th scale image is the "flaming" points drawn in ".. The "flamed parent" point $(k, l)^{(j+1)}$ is drawn in ' + '. Its corresponding four "flamed children" $\left[\begin{array}{cc}(2 k, 2 l)^{(j)} & (2 k, 2 l+1)^{(j)} \\ (2 k+1,2 l)^{(j)} & (2 k+1,2 l+1)^{(j)}\end{array}\right]$ on scale $j$ are drawn in ' $x$ '. The "unflamed 4-connection neighbors" of all the "flamed children" are the potential front on scale $j$ drawn in ' $o$ '.

Once the potential front on the $j$ th scale is determined, Fast Marching method can be applied to fast initialize the signed distance function around this front within a narrow band with speed $F=1$ outward and inward respectively. Then the obtained potential front and the signed distance function are used as the initial front condition on the $j$ th scale and a new propagation could be performed within this narrow band, as the solution must be within it.

\section{B. Fast Hermes algorithm}

Hermes algorithm is proposed by Nikos to fast implement the level set numerical solution [2]. The idea of this approach combines the Narrow Band and Fast Marching method by employing a selective propagation over a relatively small window.

A given level set partial differential equation could be rewritten as:

$$
\phi^{t+1}(x, y)=\phi^{\prime}(x, y)+\gamma\left(x, y, \phi^{\prime}\right) \delta t
$$

where $\gamma\left(x, y, \phi^{t}\right)$ is the propagation velocity. The Hermes algorithm at each step selects the pixel with the highest absolute propagation velocity and performs a local evolution to the level set function within a circular window centered on this pixel. Meanwhile, the First in, First out (FIFO) rule is used if there are several pixels with equal propagation velocity. This operation either needs to search in the heap to find the elements that have the same highest absolute velocity at each step or needs to embed the queues in the heap through the pointer that will increase the complex of the algorithm. However, this FIFO rule is not necessary. Due to the locality, the Hermes algorithm itself obtains the final solution much more rapidly by loosely respecting the constraints in the intermediate levels. In other words, it prefers to propagate some individual area much faster rather than evolve all the intermediate levels consistently. We can further loosen the constraint regardless of the FIFO rule, which yields the fast hermes algorithm. The computational cost could be greatly reduced. Build a max-heap for front sites with respect to their absolute propagation velocity such that the top element has the highest absolute velocity. Using the max-heap algorithm, fast hermes approach at each step selects the pixel on the top of the heap and performs the local updating around this pixel. The improved fast Hermes algorithm could obtain the final solution much more rapidly than the original Hermes algorithm while it could get the same final solution as that of the Hermes algorithm. 


\section{Multiscale level set evolution algorithm}

On each scale, the fast Hermes algorithm is applied to evolve the front and the solution passed from the coarser scale to the finer one is obtained by our approach proposed in section III-A.

\section{EXPERIMENTS}

Simulations have been conducted to evaluate the three algorithms discussed above. Experimental results on DSA vessels segmentation are provided. The front motion is given by the geodesic active contours equation:

$$
\frac{\partial C}{\partial t}=g_{I}\left(c_{1}+c_{2} K\right)-\left(\nabla g_{1} \bullet \vec{N}\right) \cdot \vec{N}(6)
$$

where $\vec{N}$ is the unit inward normal vector and is given by $\vec{N}=\frac{\nabla \phi}{|\nabla \phi|} . K$ is the curvature of the front and could be represented by $K=\operatorname{div}\left(\frac{\nabla \phi}{|\nabla \phi|}\right) \cdot g_{I}$, is a monotonically decreasing function such that $g_{I}(r) \longrightarrow 0$ as $r \longrightarrow \infty$ and $g_{l}(0)=1$. A common choice is given by $g_{I}=e^{-\alpha\left|\nabla G_{\sigma} * I\right|} \quad, \quad \alpha>0$, where $G_{\sigma}$ is Gussian function.

The corresponding level set equation to equation (6) is:

$\phi_{t}+g_{I}\left(c_{1}+c_{2} K\right) \cdot|\nabla \phi|-\left(\nabla g_{I} \bullet \nabla \phi\right)=0 \quad(7)$

In the following experiments, we choose $\alpha=0.2, c_{1}=-1.0, c_{2}=0.1$. The level set equation (7) is implemented by Multiscale level set evolution algorithm, fast Hermes algorithm and Hermes algorithm respectively.

Fig 2 is the segmentation results by our multiscale scheme discribed in section III-C. Fig 2(a) fig 2(d) are the front propagation on scale 1 . Its final solution (fig 2(d)) is used to determine the initial front condition on scale 0 . Fig 2 (e) shows the obtained potential front site on the finer scale by the passing solution method proposed in section III-A. In fig2 (e), the passed front is very close to the desired boundary thus can induce fast convergence on the finer scale. Fig 2 ( $f$ ) is the final segmentation map only by 600 iterations, which is obtained by using the passed solution as its initial front condition and evolving by fast Heremes algorithm. Fig 3 is the segmentation results by fast Herems algorithm discussed in section III-B and fig 4 by Hermes algorithm [2].

Comparison of fig2 fig 4 shows that the segmentation results are almost the same. However, as far the computational cost is concerned, the running time for a $128 \times 128$ DSA vessel test image using Matlab language is 18 minutes for the proposed multiscale scheme, 34 minutes for fast Hermes algorithm and 124 minutes for Hermes algorithm. Moreover, the computational time for passing the solution from the coarser scale to the finer scale is less than 0.1 second. Experiments show that fast Hermes algorithm is much faster than Hermes algorithm and the proposed multiscale scheme for the level set evolution is much faster than the monoscale fast Heremes algorithm.

Further application on medical image segmentation by our multiscale scheme is provided. Fig 5 is a pathlogical brain MR image where tumor regions are to be extracted. Fig 6 is a pulmonary vessel selected from CT image, which is preprocessed by contrast enhancement. The vessel region is to be extracted there.

The first three columns are the front propagation on scale 1. The fourth columns show the obtained potential front site on the finer scale. The fifth columns show the final front site on scale 0 . Seeing from the fourth column that the potential front are very close to the desired boundary thus the convergence on scale 0 is achieved very fast. Seeing from fig5 (e) that those tumors are extracted precisely. It could be seen from fig6 (e) that the front stops at the desired vessel boundary encouragingly, even some small and thin vessel branches, which exhibit much variability, could be located precisely. The segmentation results are very promising which show that our multiscale scheme for the level set evolution is fast and efficient.

\section{CONCLUSION}

In this paper, an efficient multiscale scheme for level set evolution was proposed. First, borrowed from the idea of the entropy condition and extended it, a new method passing the solution from the coarser scale image to the finer one was presented, where neither extrapolation nor complex computation is needed. That leads to fast convergence. Second, an improved fast Hermes algorithm was proposed to implement the level set evolution on each scale, which obtained the final solution much more rapidly by further loosening the constraint regardless of FIFO rule. Experiments on DSA vessel image segmentation show that the improved fast Hermes algorithm is much faster than the Hermes algorithm and our multiscale scheme for the level set evolution is much faster than the monoscale fast Heremes algorithm. Very promising experimental results on medical image segmentation was obtained by our approach. In the experiments, the initial front condition on the finer scale obtained by our passing solution method was very close to the final solution, which show that the proposed approach could speed up the convergence rate. In addition, the final segmentation results presented were very promising which show that our multiscale scheme for the level set evolution is very efficient and reliable.

\section{REFERENCES}

[1] J.A.Sethian, "Level Set Methods and Fast Marching Methods: Evolving interfaces in computational geometry, fluid mechanics, computer vision and material science," Cambridge University Press, 1999.

[2] N. Paragios and R. Deriche, "A PDE-Based Level Set Approach for Detection and Tracking of Moving Objects," Proc. IEEE Int"l Conf.Computer Vision, pp. 1,139-1,145, 1998. 
[3] Nikos Paragios and Rachid Deriche, "Geodesic Active Contours and Level Sets for the Detection and Tracking of Moving Objects," IEEE Transactions on pattern analysis and machine intelligence. Vol.22, No.3, March 2000

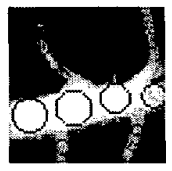

fig2(a) initial state time $=0$

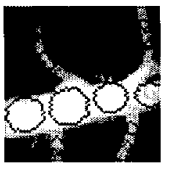

fig2(b) 60 iterations time $=1$

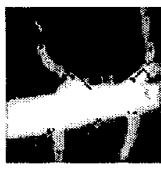

fig2(c) 1900 iterations time $=6$

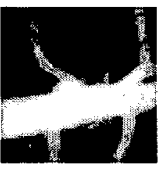

fig2(d)

2400 iterations time $=9$
[4] J.A.Sethian, "Numerical Methods for Propagating Fronts, Variational Methods for Free Surface Interfaces, " Spring-Verlag, 1987.

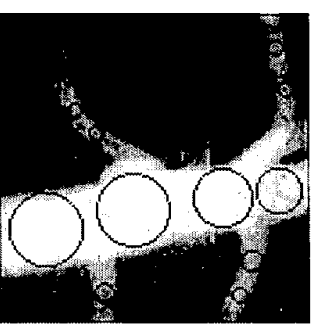

fig3(a) initial state

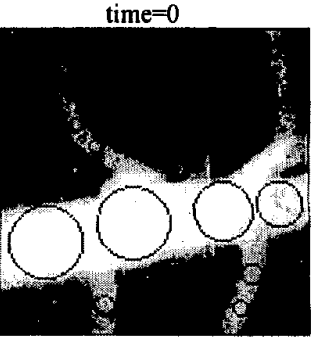

fig4(a) initial state time $=0$

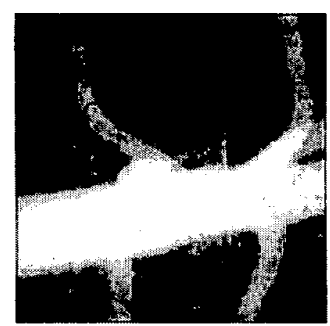

fig3(b) 5200iterations

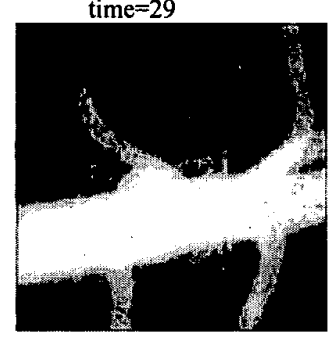

fig4(b) 5200iterations time $=97$

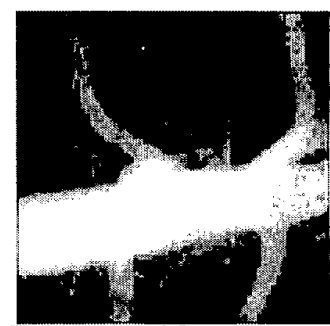

Fig2(e) passed front time $=9$

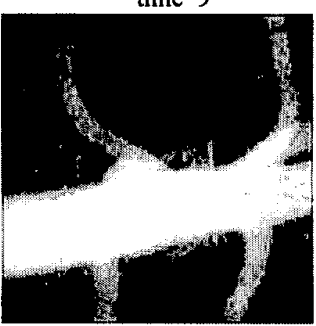

fig3(c) 6100 iterations

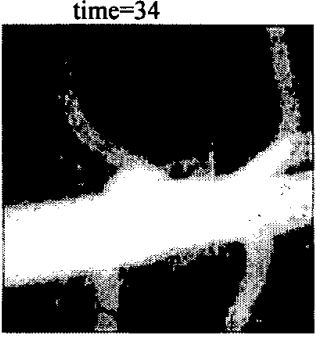

fig4(c) 6100 iterations

Fig 2 fig4 DSA segmentation by multiscale scheme, fast Hermes algorithm, Hermes algorithm respectively

Segmentation map on scalele 1

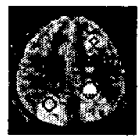

fig 5
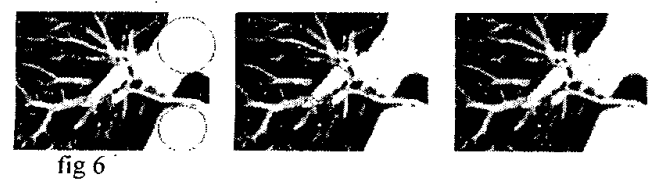

fig $5 \sim$ fig 6 medical images segmentation by multiscale level set evolution scheme
Segmentation map
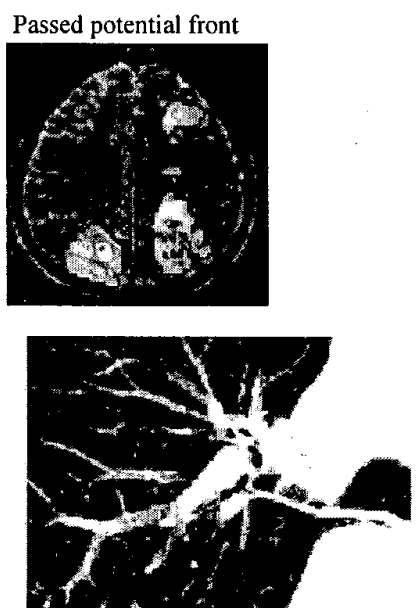

on scale 0
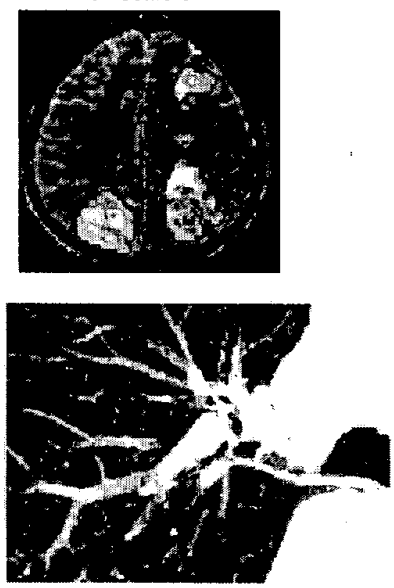